\author{
Review Article \\ www.ijrap.net
}

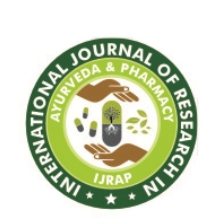

\title{
INNOVATIONS AND ADVANCEMENTS IN HERBAL DRUG STANDARDIZATION: A REVIEW
}

Saleemulla Khan ${ }^{1}$ and Aswatha Ram HN ${ }^{2} *$

${ }^{1}$ Assistant Professor Selection Grade, Department of Pharmacognosy, Manipal College of Pharmaceutical Sciences, Manipal University, Manipal, Karnataka, India

${ }^{2}$ Associate Professor, Department of Pharmacognosy, Manipal College of Pharmaceutical Sciences, Manipal University, Manipal, Karnataka, India

Received on: 10/06/16 Revised on: 12/07/16 Accepted on: 23/07/16

\author{
*Corresponding author \\ E-mail: aswatharam@gmail.com
}

DOI: $10.7897 / 2277-4343.074170$

\section{ABSTRACT}

WHO has been publishing guidelines for assessment of quality of herbal raw material from time to time, however now it is emphasizing on standardization of herbal preparations. As there are different kinds of preparations available in the traditional systems, a common technique of standardization is not available. This article reviews the recent techniques that are used in herbal drug standardization so as to find a feasible and affordable method of standardization.

Keywords: Capillary electrophoresis, Chemometrics, Combinatorial markers, Comprehensive dissolution, SCAR markers

\section{INTRODUCTION}

Since the dawn of civilization, herbs and herbo-mineral drugs have been used to treat human diseases. Herbal medicines are getting popular due to the belief that, these are cheaper and locally available remedies with fewer side effects. However, lack of standardization techniques and non-availability of marker compound sf or such traditional medicines is the only drawback of these medicines. Standardization is necessary to ensure the quality, safety and efficacy of the herbal preparations. During the past decades, efforts have been made to develop suitable standardization techniques for these herbal drugs.

Generally one or more herbs are used in herbal preparations which are considered as herbal finished herbal products. These herbal drugs may contain excipients in addition to the active ingredients. However, herbal finished products or mixed herbal product to which synthetic or chemically defined compounds or isolated constituents from herbs have been added, are not considered as herbal drugs ${ }^{8}$.

Standardization of herbal drug(s) means adjustment of a herbal preparation to a defined quantity of its constituents with a known therapeutic effect ${ }^{1}$. Standardization ensures quality, quantity and therapeutic efficacy of each dose of ingredients in a predefined manner ${ }^{2}$.Good Manufacturing Practices (GMP) implementation is another requirement for the standardization of herbal formulations ${ }^{3-5}$. Such abidance of regulations avoid batch to batch variations and minimize errors and increases the acceptance of polyherbal formulations ${ }^{6}$. The therapeutic efficacy in herbal medicines is generally attributed to the synergistic effect of the biologically active constituents which needs to be standardized ${ }^{7}$.

The traditional Ayurvedic medicine employs over 1200 different herbs and about $65 \%$ of the Indian population use herbal medicine on a regular basis. Herbal medicines are popular even in the developed countries. The Canadians $(50 \%)$ and French $(75 \%)$ have tried complementary or alternative medicine (herbal remedies) for certain ailments, according to WHO. A traditional herbal medicine, Kampo is prescribed by about $85 \%$ of medical doctors in Japan together with modern medicine. The thorny issue is standardization of herbal medicines, as they contain hundreds of chemical constituents with or without therapeutic effects may be with a little or no evidence ${ }^{9}$. Therefore this article reviews recent techniques in standardization of herbal products; a basic scheme ${ }^{10}$ of herbal drug standardization is shown in the Figure 1.

\section{Chemometrics}

In the year 1971 a Swedish scientist Svante Wold coined the term "kemometri" in Swedish which is termed as "Chemometrics" in English" ${ }^{11}$. The most commonly used methods in standardization of herbal medicines are chromatography. Though the techniques are powerful, they suffer with major drawback such as improper identification of the separated compounds. Hyphenated techniques in addition to chromatography such as LC-MS, LC-MS-MS, has paved way for better identification and clear understanding of the phytomixtures. Chemometrics further analyzes the spectroscopic data and helps in affording better chemical information. Chemometric resolution method and principal component analysis (PCA) is the commonly used technique and the scheme of instrumental data interpretation by chemometrics is presented in Figure $2^{12}$.

\section{Capillary Electrophoresis}

Because of the versatility and high separation power, Capillary electrophoresis (CE) meets the criteria for quality control of herbal drugs. A wide range of polarity and molecular weight compounds can be analysed ${ }^{13-14}$. In Capillary zone electrophoresis (CZE), separation is based on the charge to mass ratio of the analytes. Plant secondary metabolites such as Alkaloids, Phenolic acids and Flavonoids are analysed easily by CZE. Optimization of CZE methods specifically for the analysis of phytochemical compounds has been reviewed by $\mathrm{Li}$ et $a l^{15}$. 
Plant extracts containing alkaloids can be protonated easily and form water-soluble salts and the aqueous acidic electrolytes are often used as background electrolyte for CZE analysis. Hydrophobic compounds get more solubilized in non-aqueous solvents and further improve selectivity as organic solvents are widely used in CE. Aconite roots electropherogram ${ }^{16}$ is depicted in Figure 3.

Mono and polysaccharides in herbal drugs (Moutan Cortex) was standardized by applying CE. Copper working electrode was applied at a constant potential in a strong alkaline media for the analysis of carbohydrates. Paeonia suffruticosa was identified in Moutan Cortex using the markers such as paeoniflorin and paeonoside and other sugars ${ }^{17}$.

\section{Dissolution Test (Comprehensive)}

Release of active principles from the solid dosage forms is the crucial step for bioavailability and efficacy. For the quality control of oral dosage forms, active pharmaceutical ingredients are identified by dissolution studies. Dissolution study for herbal medicines is difficult due to complex nature of active principles ${ }^{18}$.In herbal medicines dissolution is limited extremely because of the poor understanding of the lead molecules characterization and their bioactivity. Moreover, factors such as origin, harvesting time and method of extraction of the herbal raw material, govern the specific formulation chemical composition to a considerable extent ${ }^{19-20}$.

Biological fate of some herbal constituents could be attributed to the bacterial biotransformation ${ }^{21}$, but still dissolution is the prerequisite for permeation to the bio membrane. Herbal solid dosage forms need to be tested for in vitro dissolution to consider the bioavailability. Previous to principal component analysis (PCA), Pareto scaling highlighted the quantitative performance by loading of peaks selectively which indicates drug release and dissolution rate of clinically implicated molecule.

For the first time, a method was developed and validated for the comprehensive dissolution test for Ginkgo biloba tablets by integration of profiling of chemical and strategy of selfreference (peak by peak). Self-reference samples chromatographic fingerprints due to the translation by PCA into chemical profiles which is the normal gross concentrations. This method was used to detect the simultaneous release of the detected constituents after the development of a HPLC fingerprint with the help of a principal component analysis and the fingerprints of Ginkgo biloba ${ }^{22}$ is represented in Figure 4.

\section{Scar Markers}

Randomly Amplified Polymeric DNA (RAPD) has been used in the identification and characterization of proteins in different biological systems. However, the techniques lack reproducibility which is a major drawback. Therefore, the technique cannot be used for the herbal drugs authentication. Other PCR and nonPCR based markers like AFLP, ISSR, SSR and RFLP have also been used for the authentication of herbal drugs. Unfortunately even these markers suffer with disadvantages like high cost, radioactive isotope use, and sequence information requirement etc.

The reproducibility of RAPD has been improved by the conversion of amplicons of RAPD into Sequence Characterized Amplified Region (SCAR) markers to make it reproducible as compared to RAPD markers alone. The method has been validated for reproducibility and hence can be safely used for the authentication of medicinal plant materials used in the preparation of traditional medicines ${ }^{23}$.

\section{Combinatorial Markers}

Selection of marker compounds may be sometimes irrelevant to the bioactivity in many cases. Therefore, Zi-Qi Shi et $a l^{24}$ proposed a universal strategy for the identification of combinatorial markers. These are bioactive and considered as chemical markers for standardization. Some common components in different batches of medicines were identified for fingerprinting and quantification. Lycoridis radiatae bulbs were used as an example and ungerimine and galanthamine were chosen as effective combinatorial markers which the authors found by fingerprint-bioactivity modeling (bioactivity and quantification of compounds) and Acetylcholinesterase inhibitory activity. This method was applied to the unknown samples also and regarded as useful for the discovery of effective combinatorial markers (ECMs) of new herbal drug candidates. The strategy ${ }^{24}$ for standardization is depicted in Figure 5.

\section{Marker Compounds}

There are three types of marker compounds ${ }^{25}$ viz., bioactive markers [e.g., vasicine, curcumin, piperine (responsible for the activity which is claimed for a particular drug)]; chemical markers [e.g., quercetin, lapachol, rutin (constituents neither specific to the drug nor to the activity of the herbal drug)], and general markers [e.g., gallic acid, lupeol (compounds distribution is wide in plants)].

\section{Marker Based Standardization}

SJT-DI-02 (marker based standardized)is a patented, novel and efficacious polyherbal formulation used for the treatment of diabetes. The formulation consists of dried extracts of Acorus calamus (rhizomes), Aegle marmelose (leaves), Benincasa hispida (fruits), Chlorophytum arendinaceum (roots), Eugenia jambolana (seeds), Ocimum sanctum (leaves), Punica granatum (pericarp), Tamarindus indica (seeds). Different fractions of extracts were mixed to prepare the formulation. The polyherbal formulation was standardized by using four different markers (marmelosin, gallic acid, lupeol and asarones). TLC system was well developed first followed by HPTLC. Quantification of marker compounds was done by TLC densitometric method. Overlay of absorption spectra was used to know the purity of bands due to the marker compounds in the extracts, and confirmed. The developed HPTLC method was found to be sensitive, reproducible and rapid $^{26}$.

Simultaneous determination of seven bioactive compounds such as ephedrine, amygdalin, liquiritin, benzoic acid, isoliquiritin, formononetin and glycyrrhizic acid was carried out in San-ao decoction (a traditional Chinese prescription) and its various combinationsusing HPLC. A validated method was adopted for the analysis briefly detection was carried out by diode array (DAD) at 210, 254 and $360 \mathrm{~nm}$; XTerra C(18) column as stationary phase and acetonitrile and a buffer solution $((0.02$ $\mathrm{mol} / \mathrm{L}$ potassium dihydrogen phosphate and adjusted to $\mathrm{pH} 3$ using phosphoric acid) as the mobile phase. The flow rate was $1.0 \mathrm{~mL} / \mathrm{min}$. A wide variation was observed in the contents of the compounds and useful in illustrating the possible drug interactions after the combination of some herbs in the different formulations ${ }^{27}$.

A high performance liquid chromatography (HPLC) method was developed and validated (ICH guidelines) for the analysis of 
ferulic acid in Asafoetida and one poly-herbal formulation. HiQSil ODS C-18 was used as the column and the mobile phase was acetonitrile: $10 \%$ acetic acid $(20: 80 \mathrm{v} / \mathrm{v})$. The method can be used for estimation of ferulic acid in formulations containing Asafoetida ${ }^{28}$.

Reverse phase high performance liquid chromatography (RPHPLC) method was developed for the estimation of trigonelline from Trigonella foenum-graecum and its marketed herbal formulations such as Dibet powder and Amyron syrup. Cosmosil CN-MS was used as the column and eluted with solvent system, methanol:distilled water [95:5, v/v; pH 3.5 using hydrochloric acid]. Photo Diode Array detector at $267 \mathrm{~nm}$ was used for the detection ${ }^{29}$.

Rapid RP-HPLC analysis of glabridin from the crude drug and herbal formulations was developed and validated. C18 column was used and the mobile phase was water and acetonitrile in a gradient elution manner with a flow rate of $1 \mathrm{~mL} / \mathrm{min} .14 .9 \pm$ $0.02 \mathrm{~min}$ was the Retention time. This method can be used for the analysis of glabridin in herbal formulations containing Licorice as one of the ingredients ${ }^{30}$.

Nardostachys jatamansi DC is used as herbal medicine singly and in multi-herbal formulations UPLC-QTOF MS, a validated method was developed for the identification of nardin in $\mathrm{N}$. jatamansi samples. Acquity BEH C(18) column was used with a gradient elution of methanol-water and $3 \mathrm{~mm}$ ammonium acetate. Negative ionization mode of QTOF mass detector was used. Authentication and quantification of the marker was done in 4 minutes using the chromatography ${ }^{31}$.

Embelin (marker) based standardization was developed and validated in plant Embelia ribes and its polyherbal formulations. Embelin was quantified by Reversed-phase high-performance liquid chromatography and coupled with diode array detection (RP-HPLC-DAD) and the method was validated. Selected phytopharmaceuticals were demonstrated using this method which can be recommended for marker based standardization and quality control of $E$. ribes containing formulations ${ }^{32}$.

Sesamum indicum seeds and its oil had been used in Ayurveda since antiquity. Lignans which are main and biologically active compounds in sesamum oil were identified as the marker compounds. A validated HPTLC method has been developed and validated for the quantification of sesamin and sesamolin (lignans) of Sesamum oil and its polyherbal formulations including the commercial ones. Densitogram followed by charring of the plate was also developed for maker based standardization of formulations containing Sesamum oil ${ }^{33}$.

A validated HPTLC method of analysis for Sennoside A and Sennoside B was developed for its quantitative analysis. Silica gel $60 \mathrm{GF}_{254}$ HPTLC plates were used in the methanolic extract analysis. The spots were visualized under UV and scanned at $350 \mathrm{~nm}$ for absorption/reflection mode. Linearity in the range of 200-1,000 ng was observed in the calibration curves.0.991 forsennoside A and 0.997 for sennoside B were the correlation coefficient. The percentage recovery was 95 and $97 \%$ respectively for Sennoside A and Sennoside B and hence a reliable and reproducible method. LOD and LOQ were found to be 0.05 and $0.25 \mu \mathrm{g} / \mathrm{g}$ respectively. They found that lot of variation in the content of Sennosides was observed from the Indian marketed poly-herbal formulations containing Senna ${ }^{34}$.

\section{Ayurvedic Formulations}

In Ayurveda, compound formulations prepared from the plants are extensively used. Plant is a mixture of compounds and desired activity may not be exhibited by one compound. Some compounds may be synergic in action or some may neutralize the possible side effects by counteracting. Therefore, it is desirable to use many plants with common anticipated activity so that the final formulation will show maximum activity in a concentrated manner ${ }^{35}$.

Ajmodadichurna is a polyherbal Ayurvedic medicine used as carminative and antispasmodic. The churna was prepared, standardized and compared with a marketed sample. Standardization was based on organoleptic characters, physical parameters and physico-chemical characteristics ${ }^{36}$.

Bhasmas (Medicated Ashes) are prepared by incineration of purified herbal extracts or minerals. Bhasmas are considered to be safe for 2 reasons; one, these formulations are not metabolized in the body and second, they maintain high alkalinity which neutralizes harmful acids. These preparations are formulated usin grasayoga, sindora, parpati, etc. However if iron, copper and sulphur are used; then the role of it component in the final products must be specified. Bhasmas being tiny particulate preparations particles measuring in nano meters, absorption and assimilation of the preparation in the body is facilitated. Therefore, bhasmas can be termed as ancient nanomedicine ${ }^{37}$.

A comparative standardization of Trivanga bhasma was carried out for In-house prepared bhasma with a marketed preparation. Evaluation was done for physical properties and chemical standardization. Fourier transform infrared spectroscopy (FTIR), Atomic absorption spectroscopy (AAS), Scanning electron microscope (SEM), X-ray Diffraction (XRD), Thermogravimetric analysis (TGA) and Atomic force microscopy (AFM) were applied for standardization. The samples were also subjected to toxicological screening and antidiabetic including diuretic activity studies. Fingerprinting of Trivanga bhasma was reported for the first time using an analytical technique ${ }^{38}$.

Brahmivati (Tablet) contains thirty eight ingredients and is used in the treatment of epilepsy and mental disorders. HPLC-UV method was developed and validated for the analysis of Bacoside A3 and Piperine which are used as the reference compounds of B. monnieri L. and P. longum L. respectively. Results showed variation in the content in marketed samples when compared with the In-house sample analysis ${ }^{39}$.

Thus, a plethora of scientific methods are available for the standardization of Ayurvedic products as of now, however Kurele $^{40}$ opines that it is not the products alone but even the premises of ASU products must be standardized as per the regulations. 


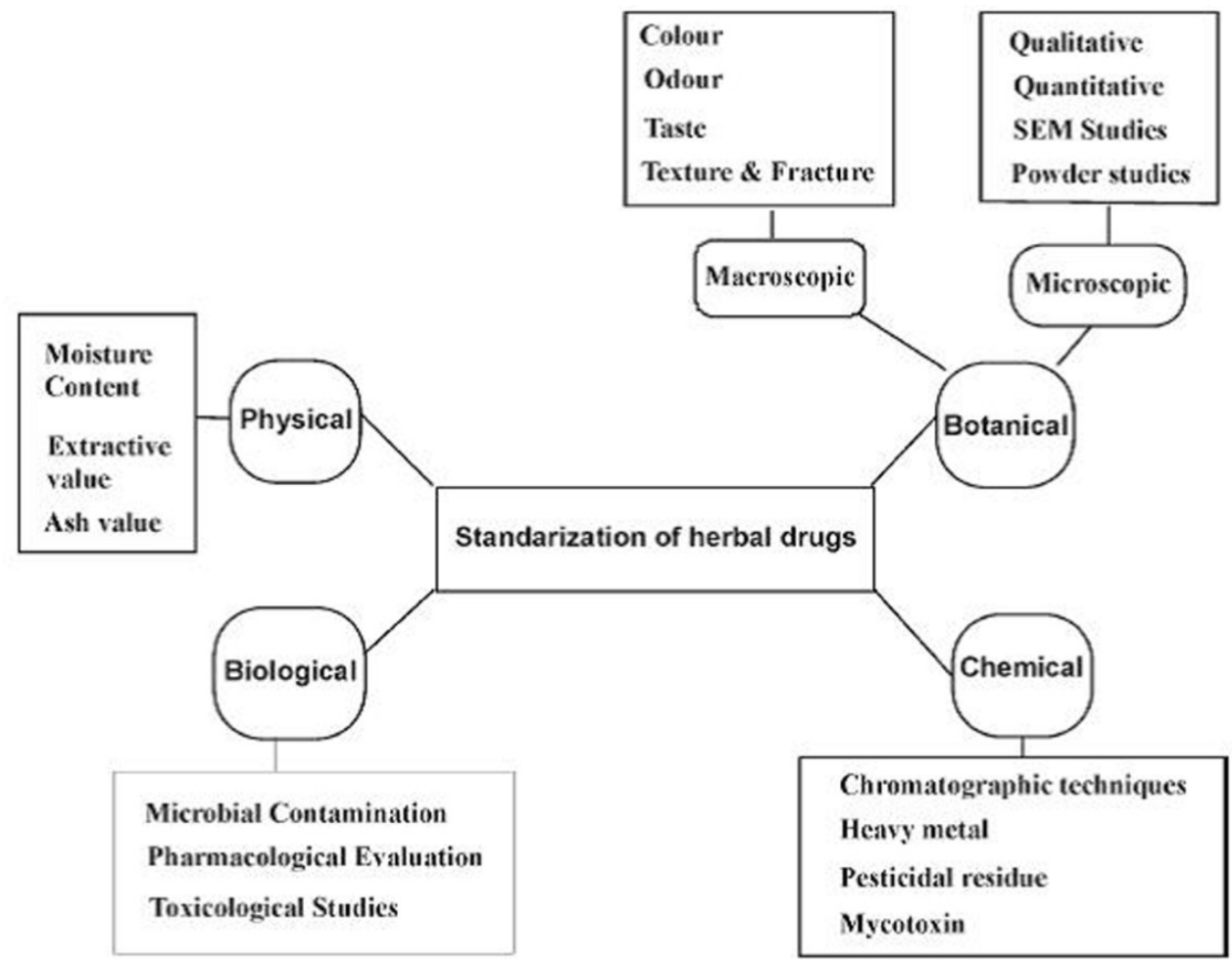

Figure 1: Herbal drug standardization scheme $\left(\right.$ Courtesy $\left.^{10}\right)$

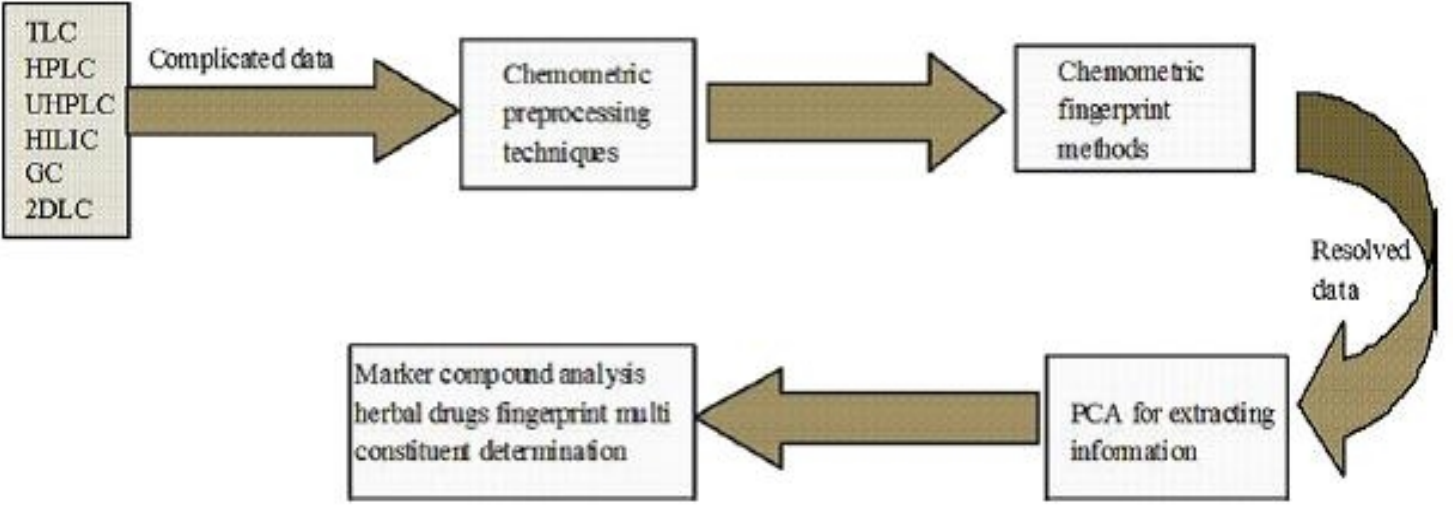

Figure 2: Scheme of interpretation of complicated data from Instruments by chemometrics. $\left(\right.$ Courtesy $\left.{ }^{12}\right)$

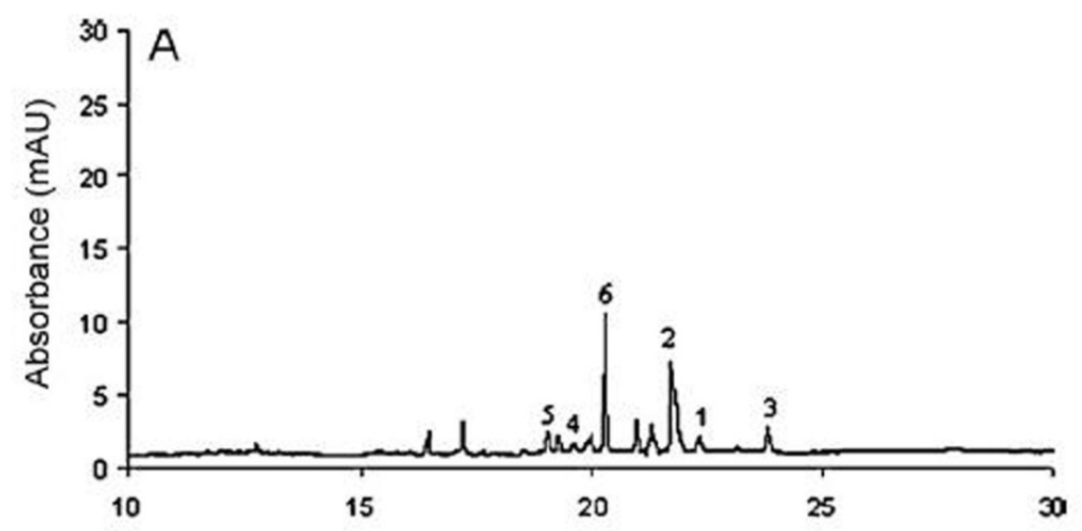

Figure 3: Typical electropherogram of Aconite roots. $\left(\right.$ Courtesy ${ }^{16}$ ) 


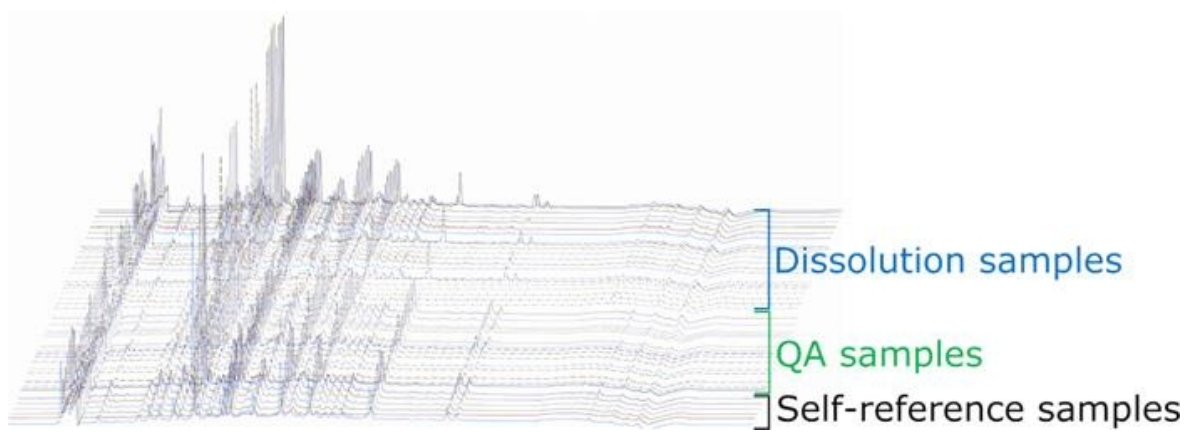

Figure 4: HPLC fingerprints of Ginkgo biloba tablets in the analytical dissolution test. (Courtesy ${ }^{22}$ )

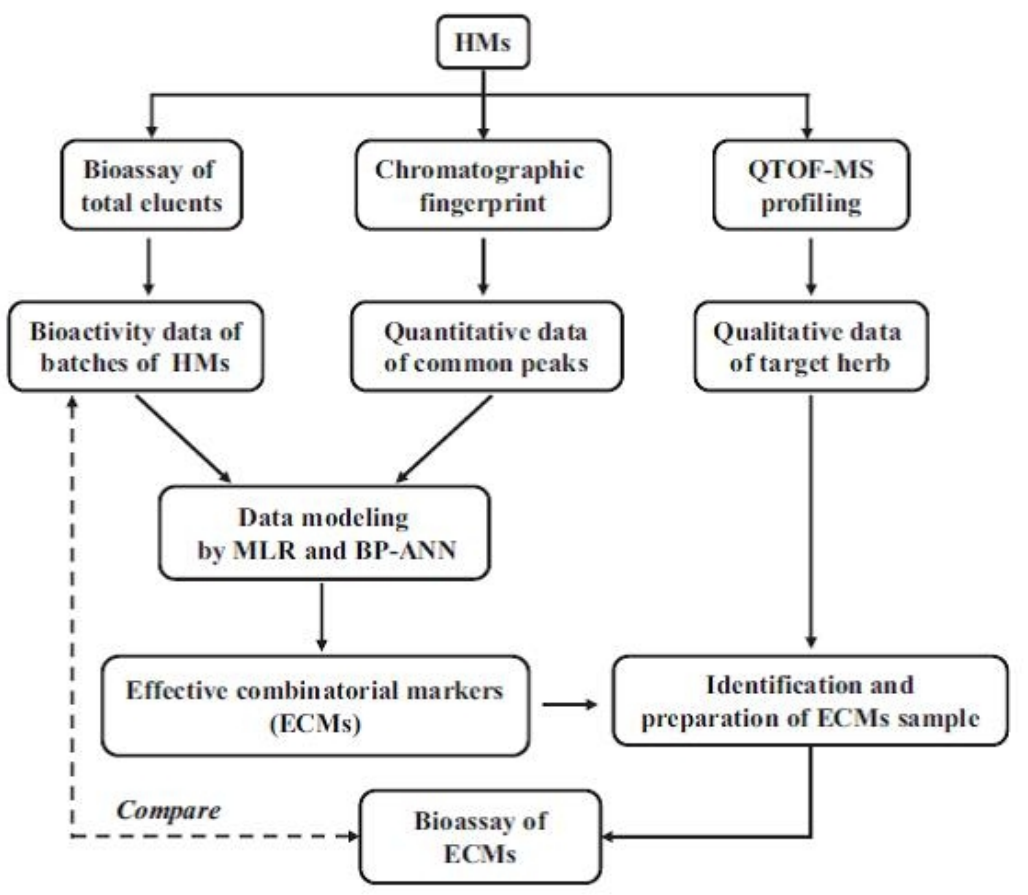

Figure 5: Standardization strategy for characterization of ECM as active markers. $\left(C_{\text {Courtesy }}{ }^{24}\right.$ )

[HM-Herbal medicines, QTOF-MS (Quadrapole-time-of-flight-Mass spectroscopy), MLR and BP-ANN are the two Chemometric methods, ECMs-Effective combinatorial markers]

\section{CONCLUSION}

Although information on standardization of herbal/ traditional medicines is plenty, yet there is a long way to go. One of the most commonly encountered problems is non-availability of marker compounds. Further, poor understanding of therapeutic activity and synergism offered by a mixture of constituents is a major hurdle in the standardization of herbal products. Efforts are being made to establish the newer and better techniques for standardization of herbal products. Application of chemometrics, capillary electrophoresis and combinatorial markers, has added new dimension to existing knowledge. This review also focuses on comprehensive dissolution techniques for herbal formulations.

\section{REFERENCES}

1. Mosihuzzaman M, Iqbal Choudhary M.Protocols on safety, efficacy, standardization, and documentation of herbal medicine (IUPAC Technical Report).Pure and Applied Chemistry 2008;80(10):2195-230.

2. Zafar R, Panwar R, SagarBhanu PS. Herbal drug standardization. The Indian Pharmacist 2005;4(36):21-25.

3. Indian Herbal Pharmacopoeia. Indian Drug Manufacturers' Association. Mumbai. 2002.

4. British Herbal Pharmacopoeia. British Herbal Medicine Association. 1996.

5. Quality Control Methods for Medicinal Plant Materials. WHO, Geneva. 1996.

6. Ahmad I, Aqil F, Owais M.Turning medicinal plants into drugs. Modern Phytomed 2006;384: 67-72.

7. Qiu J. 'Back to the future' for Chinese herbal medicines. Nat Rev Drug Discov2007;6:506-507.

8. General guidelines for methodologies on research and evaluation of traditional medicine. WHO, Geneva; $\mathrm{WHO} / \mathrm{EDM} / \mathrm{TRM} / 2000$.

9. Aschwanden C. Herbs for health, but how safe are they? Bull World Health Organ 2001; 79(7): 691-692. 
10. Neeraj Choudhary, Bhupinder Singh Sekhon. An overview of advances in the standardization of herbal drugs. J Pharm Educ Res2011;2(2):55-70.

11. Kiralj R, Ferreira M. The past, present, and future of chemometrics worldwide: some etymological, linguistic, and bibliometric investigations. J Chemometr 2006;20:247272.

12. Ankit Bansal, Vikas Chhabra, Ravindra Rawal K, Simant Sharma. Chemometrics: A new scenario in herbal drug standardization. JPharm Analysis 2014;4(4):223-233.

13. Ganzera M.Recent advancements and applications in the analysis of traditional Chinese medicines.Planta Med 2009; 75:776-783.

14. Ganzera M.Quality control of herbal medicines by capillary electrophoresis: potential, requirements and applications. Electrophoresis 2008;29:3489-3503.

15. Li P, Li SP, Wang YT. Optimization of CZE for analysis of phytochemical bioactive compounds. Electrophoresis 2006; 27:4808-4819.

16. Roberto Gotti. Capillary electrophoresis of phytochemical substances in herbal drugs and medicinal plants. J Pharm Biomed Anal 2011; 55:775-801.

17. Chen G, Zhang L, Zhu Y. Determination of glycosides and sugars in Moutan Cortex by capillary electrophoresis with electrochemical detection. J Pharm Biomed Anal 2006;41:129-134.

18. Amidon GL, Lennernas H, Shah VP, Crison JR. A theoretical basis for a biopharmaceutic drug classification: the correlation of in vitro drug product dissolution and in vivo bioavailability. Pharm Res 1995;12:413-420.

19. Lang F, Stumpf H. Considerations on future pharmacopoeial monographs for plant extracts.Pharmeuropa1999;11:268 275.

20. Westerhoff K, Kaunzinger A, Wurglics M, Dressman J, Schubert-Zsilavecz M. Biorelevant dissolution testing of St John's wort products. J Pharm Pharmacol2002;54:16151621.

21. Li H, Zhou M, Zhao A, Jia W. Traditional Chinese medicine: balancing the gut ecosystem. Phytother Res2009;23: 1332-35.

22. AnqiWanga, ZhanguoWangb, WenjunYua, Ting Zhonga, WeiyangDaia, Liang Xua, et al. Self-reference chemical profiling in the comprehensive dissolution test of herbal medicines. J Pharm Biomed Anal 2012;70:117- 125.

23. UshaKiran, Salim Khan, Khanda Jabeen Mirza, Mauji Ram, Abdin MZ.SCAR markers: A potential tool for authentication of herbal drugs. Fitoterapia 2010; 81: 969976

24. Zi-Qi Shi, De-Fang Song, Ruo-Qian Li, Hua Yang, LianWen Qi, Gui-ZhongXin, et al. Identification of effective combinatorial markers for quality standardization of herbal medicines. J Chromatography A2014;1345:78-85.

25. Rajani M,Kanaki NS.Phytochemical Standardization of Herbal Drugs and Polyherbal Formulations. In:Ramawat KG, Merillon JM. Editors.Bioactive Molecules and Medicinal Plants, Springer Science \& Business Media:2008.p.349-69.

26. Ladva BJ, Mahida VM, Kantaria UD, Gokani RH.Marker based standardization of polyherbal formulation (SJT-DI02 ) by high performance thin layer chromatography method. J Pharm Bioallied Sci2014;6(3):213-219.
27. Shu X, Tang Y, Jiang C, Shang E, Fan X, Ding A. Comparative analysis of the main bioactive components of San-ao decoction and its series of formulations. Molecules 2012;17(11):12925-12937.

28. Kareparamban JA, Nikam PH, Jadhav AP, Kadam VJ.A Validated High-performance Liquid Chromatograhy Method for Estimation of Ferulic Acid in Asafoetida and Polyherbal Preparation. Indian J Pharm Sci2013;75(4):493-495.

29. Shailajan S, Menon S, Singh A, Mhatre M, Sayed N.A validated RP-HPLC method for quantitation of trigonelline from herbal formulations containing Trigonella foenumgraecum (L.) seeds. Pharm Methods 2011;2(3):157-160.

30. Kamal YT, Singh M, Tamboli ET, Parveen R, Zaidi SM, Ahmad S. Rapid RP-HPLC method for the quantification of glabridin in crude drug and in polyherbal formulation. J Chromatogr Sci 2012;50(9):779-784.

31. Mallavadhani UV, Panigrahi R, Pattnaik B.A rapid and highly sensitive UPLC-QTOF MS method for quantitative evaluation of Nardostachys jatamansi using Nardin as the marker. Biomed Chromatogr2011;25(8):902-907.

32. Madhavan SN, Arimboor R, Arumughan C.RP-HPLC-DAD method for the estimation of embelin as marker in Embelia ribes and its polyherbal formulations. Biomed Chromatogr 2011; 25(5):600-605.

33. Sukumar D, Arimboor R, Arumughan C. HPTLC fingerprinting and quantification of lignans as markers in sesame oil and its polyherbal formulations. J Pharm Biomed Anal 2008; 5;47(4-5):795-801.

34. Aktar MW, Rajlakshmi Poi, Anjan Bhattacharyya. Status of sennosides content in various Indian herbal formulations: Method standardization by HPTLC. Bangladesh J Pharmacol 2008;3:64-68.

35. Jagetia GC, Rao SK, Baliga MS, Babu K.S. The evaluation of nitric oxide scavenging activity of certain herbal formulation in vitro: a preliminary study. Phototherapy Res 2004; 18:561-565.

36. Sriwastava NK, Shreedhara CS, Aswatha Ram HN. Standardization of Ajmodadi churna, a polyherbal formulation. Pharmacognosy Res 2010; 2(2):98-101.

37. Pal D, Sahu CK, Haldar A.Bhasma: The ancient Indian nanomedicine. J Adv Pharm Technol Res 2014;5(1):4-12.

38. Rasheed A, Naik M, Mohammed-Haneefa KP, Arun-Kumar RP, Azeem AK. Formulation, characterization and comparative evaluation of Trivangabhasma: a herbo-mineral Indian traditional medicine. Pak J Pharm Sci 2014;27(4):793-800.

39. Mishra A, Mishra AK, Tiwari OP, Jha S.HPLC analysis and standardization of Brahmivati - An Ayurvedic polyherbal formulation. J Young Pharm 2013;5(3):77-82.

40. Rajeev Kurele. Drug standardization of Ayurveda, Unani and Siddha drugs. Int. J. Res. Ayurveda Pharm. 2015;6(2):192-194 http://dx. doi.org/10.7897/22774343.06239

\section{Cite this article as:}

Saleemulla Khan and Aswatha Ram HN. Innovations and advancements in herbal drug standardization: A Review. Int. J. Res. Ayurveda Pharm. Jul - Aug 2016;7(Suppl 3):130-135 http://dx.doi.org/10.7897/2277-4343.074170

Disclaimer: IJRAP is solely owned by Moksha Publishing House - A non-profit publishing house, dedicated to publish quality research, while every effort has been taken to verify the accuracy of the content published in our Journal. IJRAP cannot accept any responsibility or liability for the site content and articles published. The views expressed in articles by our contributing authors are not necessarily those of IJRAP editor or editorial board members. 